\title{
Random Inscribing Polytopes
}

\author{
Ross M. Richardson ${ }^{1 \dagger}$, Van H. Vu${ }^{1 \dagger}$ and Lei $\mathrm{Wu}^{1 \S}$ \\ ${ }^{1}$ Department of Mathematics, University of California at San Diego, La Jolla, CA 92093-0112, USA
}

For convex bodies $K$ with $\mathcal{C}^{2}$ boundary in $\mathbb{R}^{d}$, we provide results on the volume of random polytopes with vertices chosen along the boundary of $K$ which we call random inscribing polytopes. In particular, we prove results concerning the variance and higher moments of the volume, as well as show that the random inscribing polytopes generated by the Poisson process satisfy central limit theorem.

Keywords: random polytope, inscribing, boundary, volume, variance, central limit theorem

\section{Introduction}

Let $X$ be a set in $\mathbf{R}^{d}$ and let $x_{1}, \ldots, x_{n}$ be independent random points chosen according to some distribution $\mu$ on $X$. The convex hull of the $x_{i}$ 's is called a random polytope and its study is an active area of research which links together combinatorics, geometry and probability. This study traces its root to the middle of the nineteenth century with Sylvester's famous question about the probability of four random points in the plane forming a convex quadrangle [12], and has become a mainstream research area since the mid 1960s, following the investigation of Rényi and Sulanke [11] and Efron [5].

One popular model for random polytopes is the following. Let $K$ be a convex body in $\mathbf{R}^{d}$ with volume one and $x_{1}, \ldots, x_{n}$ be independent random points chosen according to the uniform distribution on $K$. We denote this random polytope by $K_{n}$. Another one, which we call "inscribing polytopes", also begins with a convex body $K$, but the points are chosen from the surface of $K$, with respect to a properly defined measure (see [14] for the definition of such a measure). We denote this random polytope by $K_{n}^{I}$. In this paper, we assume that $K$ has smooth boundary, if not otherwise mentioned. The reader who is interested in the case of general $K$, e.g. when $K$ is a polytope, is referred to $[3,15,16]$.

The main goal of the theory of random polytopes is to understand the asymptotic behavior of key functionals on $K_{n}$, such as the volume or the number of vertices.

For most of these functionals, the expectations have been estimated (either approximately or up to a constant factor) for a long time, due to collective results of many researchers (we refer the interested reader to [1,17] and [13] for surveys). The main open question is thus to understand the distributions

\footnotetext{
†rmrichardson@math.ucsd.edu

$\ddagger_{\text {vanvu@math }}$ ucsd. edu. Research supported by a Sloan fellowship and an NSF Career Grant.

$\S$ l wu@math.ucsd.edu
} 
of these functionals around their means, as coined by Weil and Wieacker's survey from the Handbook of Geometry (see the concluding paragraph of [17])

"We finally emphasize that the results described so far give mean values hence first-order information on random sets and point processes. This is due to the geometric nature of the underlying integral geometric results. There are also some less geometric methods to obtain higher-order information or distributions, but generally the determination of variance, e.g., is a major open problem”.

The last few years have seen several developments in this direction, thanks to new methods and tools from modern probability. Let us first discuss the model $K_{n}$ where the points are chosen inside $K$. Reitzner [8], using the Efron-Stein inequality shows that

$$
\begin{gathered}
\operatorname{Var} \operatorname{Vol}\left(K_{n}\right)=O\left(n^{-\frac{d+3}{d+1}}\right), \\
\operatorname{Var} f_{i}\left(K_{n}\right)=O\left(n^{\frac{d-1}{d+1}}\right),
\end{gathered}
$$

where $f_{i}$ denotes the number of $i$-dimensional facets. Here and later the asymptotic notation is used under the assumption that $n$ goes to infinity. The hidden constants depend on $K$. Using martingale techniques, $\mathrm{Vu}[15]$ proves the following tail estimate

$$
\mathbf{P}\left(\left|\operatorname{Vol}\left(K_{n}\right)-\mathbf{E}\left(\operatorname{Vol}\left(K_{n}\right)\right)\right| \geq \sqrt{\lambda n^{-\frac{d+3}{d+1}}}\right) \leq \exp (-c \lambda)+\exp \left(-c^{\prime} n\right)
$$

for any $0<\lambda<n^{\alpha}$, where $c, c^{\prime}$, and $\alpha$ are positive constants. A similar bound also holds for $f_{i}$ with the same proof. From this tail estimate, one can deduce the above variance bound and also bounds for any fixed moments. These moment bounds are sharp, up to a constant, as shown by Reitzner in [7]. Thus, the order of magnitude of all fixed moments are determined.

Another topic where a significant development has been made is central limit theorems. It has been conjectured that the key functionals such as the volume and number of faces satisfy a central limit theorem (hereafter $C L T$ ). For instance,

Conjecture. There is a function $\epsilon(n)$ tending to zero with $n$ such that for every $x$

$$
\left|\mathbf{P}\left(\frac{\operatorname{Vol}\left(K_{n}\right)-\mathbf{E}\left(\operatorname{Vol}\left(K_{n}\right)\right)}{\sqrt{\operatorname{Var}\left(\operatorname{Vol}\left(K_{n}\right)\right)}} \leq x\right)-\Phi(x)\right| \leq \epsilon(n),
$$

where $\Phi$ denotes the distribution function of the normal distribution.

Reitzner [7], using an inequality due to Baldi and Rinott [4] (which proved a CLT for a sum of weakly dependent random variables), showed that CLT really holds for the volume and number of faces of the so-called Poisson random polytope. This is a variant of $K_{n}$, where the number of random points is not $n$, but a Poisson random variable with mean $n$. The advantage of this model is that it guarantees the independence between the number of random points in disjoint regions of $K$, which proves a significant technical advantage. Based on Reitzner's result and the tail estimate above, $\mathrm{Vu}$ [16] showed that the volumes of the Poisson random polytope and $K_{n}$ are comparable, and thus confirmed the CLT conjecture.

The above results together provide a fairly comprehensive picture about $K_{n}$. We refer the reader to the last section of [16] for a detailed summary. The main goal of this paper is to provide such a picture for the inscribing model $K_{n}^{I}$. For this model, the volume is perhaps the most interesting functional (as the 
number of vertices is always $n$ ), and thus the volume functional will be the sole focus of this work. In what follows we shall write $Y$ in place of $\operatorname{Vol}\left(K_{n}^{I}\right)$, for convenience.

The inscribing model is somewhat harder to analyze than $K_{n}$ and a sharp estimate on the volume was obtained only recently, thanks to the tremendous effort of Schütt and Werner, in a long (over one hundred pages) and highly technical paper [14]. We have

$$
\mathbf{E}(Y)=1-\left(c_{K}+o(1)\right) n^{-\frac{2}{d-1}}
$$

where $c_{K}$ is a constant depending on $K$ (the 1 here represents the volume of $K$ ). Reitzner [10] obtained estimates (which are sharp up to a constant factor) for all intrinsic volumes. He also gives an upper bound on the variance [8]:

$$
\operatorname{Var}(Y)=O\left(n^{-\frac{d+3}{d-1}}\right) .
$$

The first result we show in this paper is that the variance estimate is sharp, up to a constant factor.

\section{Theorem 1.1 (Variance)}

$$
\operatorname{Var}(Y)=\Omega\left(n^{-\frac{d+3}{d-1}}\right)
$$

The next result in this paper shows that the volume has exponential tail.

Theorem 1.2 (Concentration) For a given convex body $K$, there are constants $\alpha, c$, and $\epsilon_{0}$ such that the following holds. For any $\alpha \ln n / n<\epsilon \leq \epsilon_{0}$ and $0<\lambda \leq n$, we have

$$
\mathbf{P}\left(|Y-\mathbf{E} Y| \geq \sqrt{\lambda n^{-\frac{d+3}{d-1}}(\ln n)^{\frac{2(d+1)}{(d-1)}}}\right) \leq 2 \exp (-\lambda / 4)+\exp (-c \epsilon n) .
$$

Corollary 1.3 (Moments) For any given convex body $K$, the $k$-th moments of $Y$ satisfies

$$
M_{k}=O\left(\left(n^{-\frac{d+3}{d-1}}(\ln n)^{\frac{2(d+1)}{(d-1)}}\right)^{k / 2}\right) .
$$

Finally, we obtain the analogue of Reitzner's central limit theorem for the Poisson model. Instead of $Y$ we use $Y_{P}$ to denote the volume of the random polytope generated in this model.

Theorem 1.4 (Central Limit Theorem) $Y_{P}$ satisfies the central limit theorem.

All proofs are technical and due to space limitation, we cannot even sketch them. Let us, however, point out a key difference between $K_{n}$ and $K_{n}^{I}$. In the investigation of volume, a quantity which seems to matter much is the amount of volume change when one puts in a new random point (see [15]). In the model $K_{n}$, if we put in a new random point, it is going to effect the volume if and only if the new point falls outside the convex hull of the others. The probability of this is quite small and is related to the notion of the so-called wet-region as defined by Bárány (see [1] for a survey on methods relying on these notions). On the other hand, in the model $K_{n}^{I}$, every new point is a vertex of the convex hull and has an effect on the volume. This leads to difficulties in the analysis. For instance, we cannot yet prove the analogue of Vu's result [16] concerning the central limit theorem. 


\section{Acknowledgements}

The authors would like to thank Imre Bárány for many enlightening conversations and discussions on this subject.

\section{References}

[1] I. Bárány, Random polytopes, convex bodies, and aproximation, preprint, 2005.

[2] I. Bárány, Personal conversations, UCSD, 2005.

[3] I. Bárány, M. Reitzner, Central limit theorem for random polytopes in convex polytopes, manuscript, 2005.

[4] P. Baldi, Y. Rinott, On normal approximations of distributions in terms of dependency graphs, The Annals of Probability, Vol.17, No.4, 1646-1650, 1989.

[5] B. Efron, The convex hull of a random set of points. Biometrika, 52, 331-343, 1965.

[6] P. M. Gruber, Aspects of approximation of convex bodies, Handbook of Convex Geometry, Vol A, North-Holland/Elsevier Science Publishers, 1993. 20 3, 417-434, 2000.

[7] M. Reitzner, Central Limit Theorems for Random Polytopes, Probab. Theory Relat. Fields, to appear.

[8] M. Reitzner, Random polytopes and the Efron-Stein Jacknife inequality, Ann. Probab. 31, 2136$2166,2003$.

[9] M. Reitzner, The combinatorial structure of random polytopes. Adv. Math., 191, 178-208, 2005.

[10] M. Reitzner, Random poins on the boundary of smooth convex bodies, Transactions of the American Mathematical Society, 354 No. 6, 2243-2278, 2002.

[11] A. Rényi, R. Sulanke, Über die konvexe Hülle von $n$ zufällig gewählten Punkten. Z. Wahrsch. Verw. Geb., 2, 75-84, 1963.

[12] J. J. Sylvester, Question 1491, Educational Times, London, April, 1864.

[13] R. Schneider, Discrete aspects of stochastic geometry, Handbook of Discrete and Computational Geometry, 255-278, CRC Press, 2004.

[14] C. Schütt, E. Werner, Polytopes with vertices chosen randomly from the boundary of a convex body, Geometric Aspects of Functional Analysis 2001-2002, Srpinger Lecture Notes in Mathematics 1807, Spring-Verlag, 2003.

[15] V. H. Vu, Sharp concentration of random polytopes, to appear, GAFA, 2005.

[16] V. H. Vu, Central limit theorems for random polytopes in a smooth convex set, preprint, 2005.

[17] W. Weil and J. Wieacker, Stochastic geometry, Handbook of convex geometry, Vol. A, B, 13911438, North-Holland, Amsterdam, 1993. 